\title{
Distinct, crucial roles of flavonoids during legume nodulation
}

\author{
Senthil Subramanian ${ }^{1}$, Gary Stacey ${ }^{2}$ and Oliver $\mathrm{Yu}^{1}$ \\ ${ }^{1}$ Donald Danforth Plant Science Center, 975 North Warson Road, Saint Louis, MO 63132, USA \\ ${ }^{2}$ National Center for Soybean Biotechnology, Divisions of Plant Science and Biochemistry, Department of Molecular Microbiology \\ and Immunology, Christopher S. Bond Life Sciences Center, University of Missouri, Columbia, MO 65211, USA
}

\begin{abstract}
RNA interference-mediated silencing of the key flavonoid and isoflavone biosynthesis enzyme, respectively, by two different research groups has provided direct genetic evidence for the essential roles that these compounds play in nodulation. Anton Wasson et al. have shown that flavonoids are essential for localized auxin transport inhibition during nodulation in the indeterminate legume Medicago truncatula. By contrast, Senthil Subramanian et al. have shown that isoflavones are essential for endogenous nod gene induction in the determinate legume soybean.
\end{abstract}

\section{Role of flavonoids in nodulation}

Flavonoids are a group of ubiquitous and diverse molecules produced via the phenylpropanoid pathway in higher plants (Box 1). They have diversified in form and function with the evolution of land plants. Flavonoid biosynthesis occurs in most plant parts; flavonoids play a variety of roles in plants including protection against UV damage and pathogenic microbes, acting as pigments or co-pigments in influencing flower color, modulating auxin distribution, and as signal molecules to symbiotic microbes [1].

Legumes form a symbiotic association with nitrogenfixing soil bacteria called rhizobia. This symbiosis results in the formation of root nodules where biological nitrogen fixation occurs. The role of flavonoids in nodulation is intriguing because it is possible that these compounds might play multiple roles during the process. Legumerhizobial nodulation begins with signal exchange between the symbiotic partners. This is initiated by flavonoid and isoflavonoid compounds exuded through the plant roots [2]. The rhizobial symbionts recognize specific flavonoid signals produced by compatible host legumes and respond by producing a novel, lipo-chitooligosaccharide signal (Nod signal). The type of flavonoid molecules secreted by the plant and the ability of the rhizobial species to recognize these molecules, and then induce Nod signal biosynthesis is the earliest step in determining host-specificity [3]. On the roots of a compatible host plant, these bacterial Nod signals induce a series of physiological and developmental responses leading to the formation of a functional nodule. One of these responses is cell division in cortex cells to initiate the nodule primordium. In some legumes, the primordial cell division is preceded by an inhibition in

Corresponding author: Yu, O. (oyu@danforthcenter.org).

Available online 25 June 2007. auxin transport caused by flavonoids. Thus, flavonoids might play a crucial role as auxin transport regulators during nodulation [4]. The third role that flavonoids play during nodulation is to act as Nod signal inducers inside the plant roots. This secondary induction of Nod signals is thought to be responsible for an additional level of host specificity $[5,6]$. Thus, flavonoids might be playing a multitude of roles during the process of nodulation. However, until recently, most evidence implicating flavonoids in nodulation was based on indirect assays (see below).

\section{Flavonoids are essential for nodulation}

For several decades, flavonoids were largely presumed to function solely as inducers of rhizobial nod gene expression (nod genes are associated with Nod signal biosynthesis in rhizobia) and as chemo-attractants to concentrate the compatible rhizobium at the root surface $[7,8]$. However, these roles were based largely on in vitro studies. For example, exogenous addition of isoflavones was shown to induce the expression of nod-lacZ fusions in Bradyrhizobium japonicum [9]. Such studies, although strongly suggestive, were taken as dogma with no unequivocal support for an essential role for flavonoids in the nodulation process. Even though flavonoids were the most potent nod gene inducers, other non-flavonoid compounds such as betaines and xanthones were also able to induce nod genes in rhizobia [10]. Specifically, no genetic evidence existed to suggest a definitive role for flavonoids in nodulation, mostly because altering flavonoid profiles of a legume species was technically challenging.

Recent advances in the understanding of flavonoid biosynthesis, and the development of efficient hairy root transformation techniques, allowed the investigation of the long-standing question regarding the role of flavonoids in nodulation: are flavonoids essential for nodulation? To examine the role of flavonoids in the nodulation process, Anton Wasson et al. [11] used Agrobacterium rhizogenesmediated hairy root transformation to silence the chalcone synthase gene (CHS; Box 1), encoding the entry point enzyme of flavonoid biosynthesis in M. truncatula. Flavones, a group of flavonoids, were shown to be the most potent inducers of nod genes in Sinorhizobium meliloti, which colonizes $M$. truncatula. Using a similar RNAimediated approach, Senthil Subramanian et al. [12] silenced isoflavone synthase (IFS; Box 1), the entry point enzyme for isoflavone biosynthesis in soybean. Isoflavones are the major nod gene inducers of Bradyrhizobium 


\section{Box 1. The phenylpropanoid pathway}

A partial diagram of the phenylpropanoid pathway showing intermediates in flavonoid biosynthesis (Figure I). Chalcone synthase (CHS) activity creates the 15 carbon backbone of flavonoid compounds and several derivatives are produced via different, usually competing, branch pathways. Isoflavone synthase is the primary enzyme responsible for isoflavone biosynthesis. The pathway present in all higher plants is indicated by blue arrows; the isoflavonoid-specific pathway is indicated by green arrows.

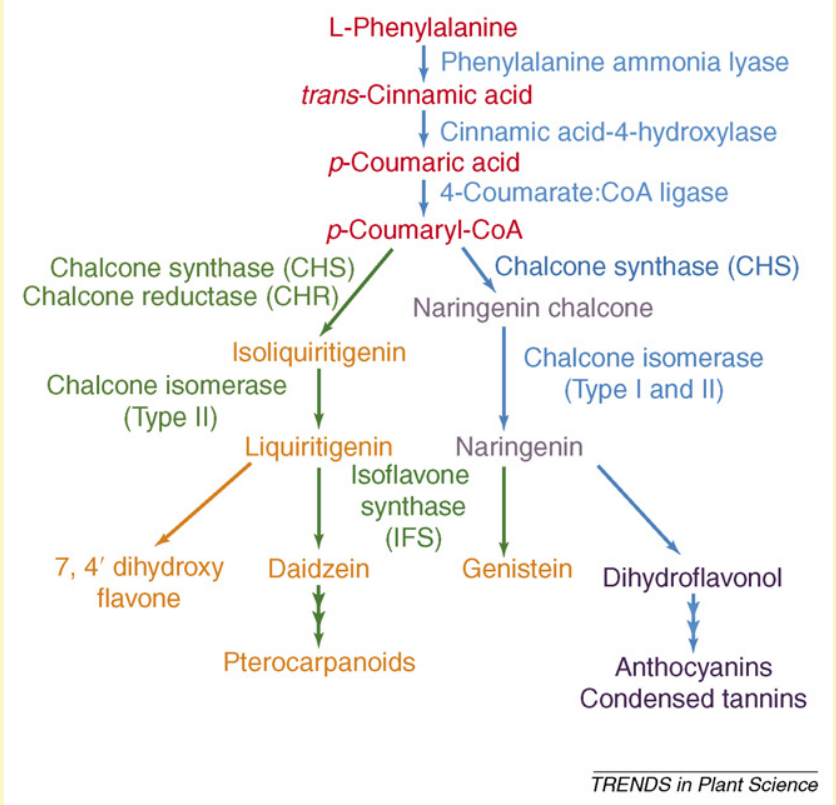

Figure I.

japonicum, the major symbiont of soybean. Essentially no flavonoid and isoflavonoid compounds were detected in the CHS RNAi $M$. truncatula roots, and isoflavonoids were virtually undetectable in the IFS RNAi soybean roots. In both cases, there was a major reduction in nodulation compared with the nodulation in control roots. The soybean data also suggested that the extent of nodulation was directly influenced by the residual isoflavone levels. These studies established for the first time that flavonoids are indispensable for nodulation.

\section{Flavonoids play a crucial role inside the plant root during nodulation}

As mentioned before, flavonoids can play multiple roles at different stages of nodulation. Flavonoids are thought to serve as signal molecules in the rhizosphere to concentrate compatible rhizobia and induce Nod signal biosynthesis. In the presence of a compatible bacterium, plant root hairs deform and curl to entrap the bacteria. The bacteria gain access to deeper cell layers via tubular plant-derived structures termed infection threads. These initial responses of nodulation were observed in both $M$. truncatula and soybean roots depleted of flavonoids. Perhaps non-specific nod gene induction by flavonoid precursors and non-flavonoid compounds (e.g. betaines, xanthones) in the exudates was sufficient to initiate this response. This would suggest that specific nod gene-inducing flavonoids do not play a crucial role during the initial stages of nodulation when the rhizobia are on the root surface. Pretreatment of rhizobial cells with the appropriate nod gene-inducing flavonoid did not restore nodulation in flavonoid-silenced roots, suggesting that nod gene induction in the rhizosphere is not sufficient for nodulation. These and other experiments strongly suggested that the flavonoids play a crucial role in nodulation once the bacteria enter the plant and exogenous flavonoids are no longer available.

A major role thought to be played by flavonoids once the bacteria enter the plant is the modulation of auxin transport during the initiation of nodule primordia. There is extensive evidence available for the role of flavonoids in regulating auxin transport. However, most of this stems from genetic studies in Arabidopsis, which is a non-nodulating species. In the flavonoid-null mutants of Arabidopsis, there is an increased level of root auxin transport compared with that in the wild type and this phenotype can be complemented by external application of flavonols. Genetic studies not only identified two protein complexes that might bind to flavonoids and regulate polar auxin transport (AtAPM and AtMDR), but also discovered that the most likely route of interaction between flavonoids and auxin transporters is through Pinformed gene (PIN) homologs [13]. In vitro studies have shown that flavonoids affect polar auxin transport in a similar way to the synthetic chemical inhibitor 1-naphthylphthalamic acid (NPA) [1].

Auxin accumulation and transport during nodulation have been studied primarily using auxin-inducible reporter gene constructs $[4,14]$. Auxin homeostasis during nodulation appears to be different between the two types of legume nodules. Legume nodules are classified into determinate and indeterminate nodules based on their ontogeny. Determinate nodules are spherical, arise from root outer cortical cells and do not contain a persistent meristem. Examples of plants producing determinate nodules include tropical and subtropical legumes such as soybean (Glycine max), cowpea (Vigna unguiculata), common bean (Phaseolus vulgaris) and Lotus japonicus. By contrast, indeterminate nodules are cylindrical, arise from root pericycle and inner cortical cells, and contain a persistent terminal meristem. Examples of plants producing indeterminate nodules include temperate legumes such as alfalfa (Medicago sativa), pea (Pisum sativum), white clover (Trifolium repens), and $M$. truncatula. Determinate nodules grow primarily by cell expansion owing to the lack of a persistent meristem and indeterminate nodules grow primarily by cell division at the terminal meristem. Earlier reviews provide a wealth of information about the ontogeny, structure and evolutionary differences between the two types of nodules $[15,16]$.

\section{Flavonoid-regulated auxin transport inhibition can be crucial during indeterminate nodule formation}

In the roots of indeterminate nodule-forming plants such as white clover and vetch, an absence of the auxin-inducible reporter gene expression was observed below the site of rhizobial inoculation $[4,17]$. This suggested an inhibition in auxin transport at the site of rhizobial inoculation. Using radioactive auxin ${ }^{3} \mathrm{H}$-IAA as tracer, Wasson et al. [11] measured auxin transport during nodulation in M. truncatula. Their results established that an inhibition 
in auxin transport did indeed occur at the site of rhizobial inoculation. RNAi silencing of flavonoid biosynthesis in M. truncatula led to increased auxin transport in these composite plant hairy roots providing genetic evidence that flavonoids do act as auxin transport inhibitors in this species. Flavonoid-deficient roots lacked the ability to inhibit auxin transport at the site of rhizobial inoculation whereas control roots showed a clear inhibition in auxin transport. The lack of nodules on these roots in response to rhizobial inoculation strongly suggests that the ability to inhibit auxin transport in response to rhizobial inoculation is essential for nodulation in this species.

\section{Flavonoid-regulated auxin transport inhibition is not crucial during determinate nodule formation}

In the roots of determinate nodule-forming plants L. japonicus and soybean, the expression of auxin-inducible $\beta$-glucuronidase (GUS) at the site of inoculation was not significantly altered by infection with respective rhizobia $[12,14]$. RNAi silencing of isoflavone biosynthesis in soybean, also led to increased auxin transport in composite plant hairy roots suggesting that isoflavones act as auxintransport inhibitors in soybean. This was corroborated by the observation that exogenous application of the isoflavone genistein inhibited auxin transport in wild-type soybean seedlings [12]. Similar to the situation in $M$. truncatula, flavonoid-depleted soybean roots failed to nodulate, when inoculated with $B$. japonicum, suggesting that isoflavone-mediated auxin transport might be crucial to nodulation in soybean. Given that isoflavones are the major nod gene inducers in this symbiosis, the possibility existed that isoflavones could be essential to the symbiosis either because of their role as nod gene inducers and/or because of their role in inhibiting polar auxin transport. However, feeding the isoflavone genistein back to the IFS RNAi roots was not sufficient to restore nodulation, suggesting tissue-specific accumulation of isoflavones is more important than the total root isoflavone levels. More importantly, inoculation of soybean plants silenced for IFS expression with a genistein-hypersensitive $B$. japonicum mutant resulted in normal nodulation. This $B$. japonicum mutant is able to synthesize the Nod signal in the presence of low levels of isoflavone nod gene inducers [18]. These data clearly indicate that the essential role played by isoflavones in soybean nodulation is related to their role in nod gene induction. Clearly, isoflavones modulate polar auxin transport in soybean roots. However, either this response is not essential or redundant functions (perhaps from other flavonoids) allow nodulation to occur even when isoflavone levels are undetectable. Consistent with these results, using radioactive auxin ${ }^{3} \mathrm{H}-\mathrm{IAA}$ as a tracer, no measurable inhibition of local auxin transport occurred at the site of rhizobial inoculation in soybean roots (S. Subramanian et al., unpublished). Thus, isoflavone-mediated modulation of local auxin transport at the site of rhizobial infection is not crucial during determinate nodulation of soybean. However, such a role remains possible in the case of indeterminate nodulating plants such as $M$. truncatula (Figure 1). Is there sustained Nod signal biosynthesis after the bacteria enter the plant roots? Perhaps, yes. It was recently reported that plant Nod signal receptors are expressed in deeper layers of the root, suggesting that sustained expression of the rhizobial nod genes (encoding Nod signal synthesis) is required inside the root $[5,6]$. This would necessitate the presence of nod gene inducers (flavonoids) in deeper root cell layers. (a) Indeterminate nodule initiation

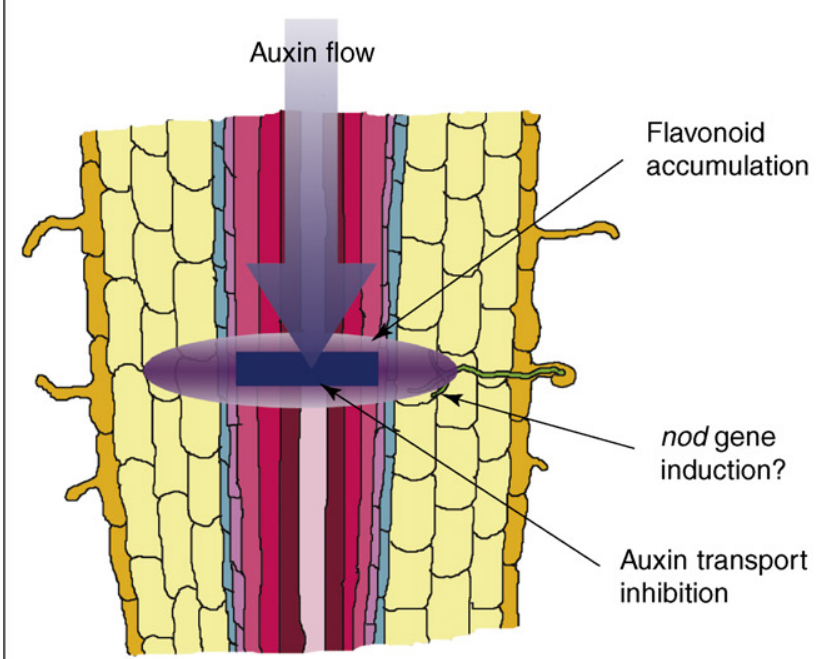

(b) Determinate nodule initation

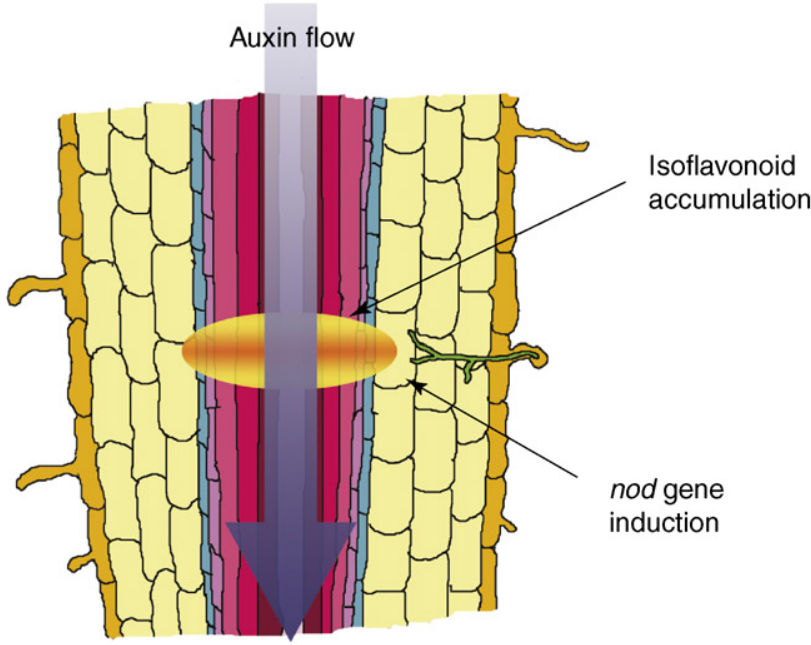

Figure 1. Flavonoids play a crucial role during nodulation after the rhizobia have entered the roots. The two possible roles for flavonoids are to inhibit auxin transport at the site of rhizobial infection (a) or to induce rhizobial Nod signal biosynthesis (b). Flavonoid-depleted roots of the indeterminate nodule-forming legume Medicago truncatula lack auxin transport inhibition at the site of rhizobial infection and have significantly reduced nodulation [11]. Isoflavonoid-depleted roots of the determinate nodule-

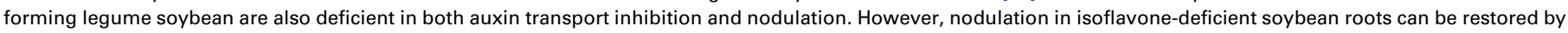
using isoflavone-hypersensitive rhizobia cells, suggesting that induction of Nod signal biosynthesis by isoflavones is crucial, whereas auxin transport inhibition by isoflavones is not [12]. In M. truncatula, flavonoids could be essential for both these processes. Consistently, during indeterminate nodule formation, flavonoid-regulated auxin transport inhibition occurs at the site of rhizobial infection (a), whereas during determinate nodule formation there is no significant auxin transport inhibition (b). 


\section{Acknowledgements}

Research in the Yu laboratory was supported by grants from the National Science Foundation (MCB0519634), USDA (NRI2005-05190), and MSMC (Grant 02-242). The Stacey laboratory was supported by a grant (DBI-0421620) from the National Science Foundation, Plant Genome program.

\section{References}

1 Taylor, L.P. and Grotewold, E. (2005) Flavonoids as developmental regulators. Curr. Opin. Plant Biol. 8, 317-323

2 Peters, N.K. et al. (1986) A plant flavone, luteolin, induces expression of Rhizobium meliloti nodulation genes. Science 233, 977-980

3 Fisher, R.F. and Long, S.R. (1992) Rhizobium-plant signal exchange. Nature 357, 655-660

4 Mathesius, U. et al. (1998) Auxin transport inhibition precedes root nodule formation in white clover roots and is regulated by flavonoids and derivatives of chitin oligosaccharides. Plant J. 14, 23-34

5 Madsen, E.B. et al. (2003) A receptor kinase gene of the LysM type is involved in legume perception of rhizobial signals. Nature 425, 637-640

6 Parniske, M. and Downie, J.A. (2003) Plant biology: locks, keys and symbioses. Nature 425, 569-570

7 Rolfe, B.G. (1988) Flavones and isoflavones as inducing substances of legume nodulation. Biofactors 1, 3-10

8 Stougaard, J. (2000) Regulators and regulation of legume root nodule development. Plant Physiol. 124, 531-540

9 Banfalvi, Z. et al. (1988) Regulation of nod gene expression in Bradyrhizobium japonicum. Mol. Gen. Genet. 214, 420-424
10 Phillips, D.A. et al. (1992) Trigonelline and stachydrine released from alfalfa seeds activate Nodd2 protein in Rhizobium meliloti. Plant Physiol. 99, 1526-1531

11 Wasson, A.P. et al. (2006) Silencing the flavonoid pathway in Medicago truncatula inhibits root nodule formation and prevents auxin transport regulation by rhizobia. Plant Cell 18, 1617-1629

12 Subramanian, S. et al. (2006) Endogenous isoflavones are essential for the establishment of symbiosis between soybean and Bradyrhizobium japonicum. Plant J. 48, 261-273

13 Peer, W.A. et al. (2004) Variation in expression and protein localization of the PIN family of auxin efflux facilitator proteins in flavonoid mutants with altered auxin transport in Arabidopsis thaliana. Plant Cell 16, 1898-1911

14 Pacios-Bras, C. et al. (2003) Auxin distribution in Lotus japonicus during root nodule development. Plant Mol. Biol. 52, 1169-1180

15 Doyle, J.J. (1998) Phylogenetic perspectives on nodulation: evolving views of plants and symbiotic bacteria. Trends Plant Sci. 3, 473-478

16 Hirsch, A.M. (1992) Developmental biology of legume nodulation. New Phytol. 122, 211-237

17 Boot, K.J. et al. (1999) Lipochitin oligosaccharides from Rhizobium leguminosauram bv. viciae reduce auxin transport capacity in Vicia sativa subsp. nigra roots. Mol. Plant Microbe Interact. 12, 839-844

18 Ip, H. et al. (2001) Bradyrhizobium japonicum mutants with enhanced sensitivity to genistein resulting in altered nod gene regulation. Mol. Plant Microbe Interact. 14, 1404-1410

1360-1385/\$ - see front matter ๔ 2007 Elsevier Ltd. All rights reserved.

doi:10.1016/j.tplants.2007.06.006

\title{
Response to Alpi et al.: Plant neurobiology: the gain is more than the name
}

\section{Eric D. Brenner ${ }^{1}$, Rainer Stahlberg ${ }^{2}$, Stefano Mancuso ${ }^{3,4}$, František Baluška ${ }^{4,5,6}$ and Elizabeth Van Volkenburgh ${ }^{2}$}

\author{
${ }^{1}$ Genomics, New York Botanical Garden, NY 10458, USA \\ ${ }^{2}$ Department of Biology, University of Washington, Seattle, WA 98195, USA \\ ${ }^{3}$ Department of Horticulture, University of Florence, Viale delle Idee 30, 50019 Sesto Fiorentino (FI), Italy \\ ${ }^{4}$ International Plant Neurobiology Laboratory, Viale delle idee 30, 50019 Florence, Italy; Kirschallee 1, 53115 Bonn, Germany \\ ${ }^{5}$ Institite of Cellular and Molecular Botany, Rheinische Friedrich-Wilhelms-University of Bonn, Kirschallee 1, 53115 Bonn, Germany \\ ${ }^{6}$ Institute of Botany, Slovak Academy of Sciences, Dubravska cesta 14, SK-84223, Bratislava, Slovak Republic
}

The past three years have seen three thought-provoking and well attended symposia, the founding of a new society, the Society of Plant Neurobiology (SPN), and the promising start of a new journal, Plant Signaling and Behavior. Although most participants within the Plant Neurobiology framework are finding plenty of stimulating, inspirational and controversial material, other colleagues have some concern about the scientific validity and rationale behind 'plant neurobiology'. Vigilance is a justifiable concern of the scientific community. In their critique of Plant Neurobiology, Amedeo Alpi et al. [1] ask the question "What longterm scientific benefits will the plant science research community gain from the concept of 'plant neurobiology?", which they believe is ' . . based on superficial analogies and

DOI of original article: 10.1016/j.tplants.2007.03.002

Corresponding author: Brenner, E.D. (ebrenner@nybg.org);

Baluška, F. (baluska@uni-bonn.de);Van Volkenburgh,E. (lizvanv@u.washington.edu).

Available online 25 June 2007. questionable extrapolations...' such as nerves, brain synapses, intelligent responses being expatriated from the field of animal neurobiology to explain some of the complex behavior of plants.

Most of our statements and publications should have made clear that plant neurobiology is pursuing a framework of ideas that were introduced by outstanding representatives of the plant sciences such as Wilhelm Pfeffer [2,3], Charles Darwin [4], Julius von Sachs [5], Georg Haberlandt [6] and Erwin Bünning [7]. No one proposes that we literally look for a walnut-shaped little brain in the root or shoot tip or some myelinated superconducting nerve cells in plants. Neither did Haberlandt [6] when he compared long-distance signalling in Mimosa with that in animals, nor Darwin when he considered the Venus' flytrap as the most animal-like plant [8] or conjectured that the root tip fulfills complex tasks like a brain [4]. 